\title{
A simple robust estimation for parameters of Cauchy distribution
}

\author{
Sándor Fegyverneki
}




\title{
A SIMPLE ROBUST ESTIMATION FOR PARAMETERS OF CAUCHY DISTRIBUTION
}

\author{
S. FEGYVERNEKI
}

Received 17 June, 2013

\begin{abstract}
We define robust estimators for the parameters of the Cauchy distribution based on the probability integral method. The proposed estimators have bounded influence functions and high breakdown points. These estimators are simple, robust and consistent, but asymptotically less efficient than the maximum likelihood estimators which are not robust. A simulation study for finite sample size shows that the efficiency of these robust estimators is rather similar to the maximum likelihood ones.
\end{abstract}

2010 Mathematics Subject Classification: 62E20; 62G35; 61E37

Keywords: M-estimates, robustness, Cauchy distributions

\section{INTRODUCTION}

Let $\left\{\xi_{i}, 1 \leq i \leq n\right\}$ be an i.i.d. sequence of random variables from the Cauchy distribution

$$
G_{0}\left(\frac{x-\mu}{\sigma}\right)=\frac{1}{2}+\frac{1}{\pi} \tan ^{-1}\left(\frac{x-\mu}{\sigma}\right)
$$

where $\sigma>0$ (see [8],[9],[10]).

The estimation of the parameters is a well discussed problem. Mostly, the location parameter $\mu$ and the scale parameter $\sigma$ are estimated by the maximum likelihood (ML) estimators $\hat{\mu}$ and $\hat{\sigma}$, respectively. However, the ML estimators are conditional robust and the breakdown points are low. There exists other estimators, which are rather efficient or even unbiased, as linear estimators of order statistics (median, median absolute deviation).

In [2], [7], [9] Csernyák, Nagy and Steiner made an analysis how the I-divergence can be used to provide simple, efficient and robust estimator for parameters.

The aim of this short note is to make an analysis the probability integral transform estimators for the parameters $\mu$ and $\sigma$ of the Cauchy distribution $G_{0}$. We know that if the random variable $\xi$ has strictly monotone increasing probability distribution

This research was (partially) carried out in the framework of the Center of Excellence of Mechatronics and Logistics at the University of Miskolc. 
function $F$ then the random variable $F(\xi)$ has uniform distribution on the interval $[0,1]$. Therefore

$$
\begin{gathered}
\sum_{i=1}^{n}\left(G_{0}\left(\frac{\xi_{i}-\mu}{\sigma}\right)-\frac{1}{2}\right)=0 \\
\sum_{i=1}^{n}\left(\left(G_{0}\left(\frac{\xi_{i}-\mu}{\sigma}\right)-\frac{1}{2}\right)^{2}-\frac{1}{12}\right)=0 .
\end{gathered}
$$

When the solutions $T_{n}$ and $s_{n}$ of this system of equations exist, $T_{n}$ and $s_{n}$ are called the probability integral transformation (PIT) estimators of the location and the scale parameter, respectively. These estimators are so-called $M$-estimators which are well-known in the context of robust estimation (see [3], [4], [5], [6]).

\section{MAIN RESULTS}

Theorem 1. Assume that $G_{0}$ is differentiable, strictly monotone increasing, then $T_{n}$ and $s_{n}$ are well defined, that is, (1.2) has a unique solution with $s_{n}>0$.

The main steps of the proof: We follow [6]. The Jacobian of the map

$$
(t, s) \longrightarrow\left(\int \psi\left(\frac{x-t}{s}\right) F(d x), \int \chi\left(\frac{x-t}{s}\right) F(d x)\right)
$$

is

$$
-\frac{1}{s}\left(\begin{array}{cc}
\int \psi^{\prime}(y) d F & \int y \psi^{\prime}(y) d F \\
\int \chi^{\prime}(y) d F & \int y \chi^{\prime}(y) d F
\end{array}\right)
$$

with $y=\frac{x-t}{s} . F$ is indifferently either the true or the empirical distribution.

We define a new probability measure $F^{\star}$ by

$$
F^{\star}(d y)=\frac{\psi^{\prime}(y)}{E_{F}\left(\psi^{\prime}(y)\right)} F(d x)
$$

then The Jacobian can be written as

$$
-\frac{1}{s} E_{F}\left(\psi^{\prime}(y)\right)\left(\begin{array}{cc}
1 & E_{F^{\star}}(y) \\
E_{F^{\star}}\left(\frac{\chi^{\prime}}{\psi^{\prime}}\right) & E_{F^{\star}}\left(y \frac{\chi^{\prime}}{\psi^{\prime}}\right)
\end{array}\right)
$$

Its determinant

$$
\left[\frac{E_{F}\left(\psi^{\prime}(y)\right)}{s}\right]^{2} \operatorname{cov}_{F^{\star}}\left(y, \frac{\chi^{\prime}}{\psi^{\prime}}\right)
$$

is strictly positive. The existence of a solution now follows from the observation that for each fixed $s$, the first component of the map has a unique zero at some $t=t(s)$ that depends continuously on $s$. We now conclude from the intermediate value theorem for continuous functions that the solution exists uniquely. The joint asymptotic distribution of $\left(T_{n}, s_{n}\right)$ can be derived from a general result of Boos and Serfling [1]. 
Theorem 2. The joint distribution of $\left(T_{n}, s_{n}\right)$ converges to a normal one:

$$
\sqrt{n}\left(\left(T_{n}, s_{n}\right)-(\mu, \sigma)\right) \stackrel{d}{\longrightarrow} N(0, \Sigma)
$$

where the covariance matrix $\Sigma$ is given by

$$
\Sigma=\sigma^{2}\left(\begin{array}{cc}
\frac{\pi^{2}}{3} & 0 \\
0 & \frac{\pi^{4}}{45}
\end{array}\right)
$$

that is,

$$
\Sigma \approx \sigma^{2}\left(\begin{array}{cc}
3.289868134 & 0 \\
0 & 2.164646468
\end{array}\right) .
$$

The main steps of the proof: We use the general result of Boos and Serfling [1] on the central limit theorem for $\left(T_{n}, s_{n}\right)$ for proving this theorem. $T_{n}$ and $s_{n}$ are the solutions of

$$
\sum_{i=1}^{n} \psi\left(\frac{\xi_{i}-\mu}{\sigma}\right)=0, \quad \sum_{i=1}^{n} \chi\left(\frac{\xi_{i}-\mu}{\sigma}\right)=0
$$

where

$$
\psi(x)=G_{0}(x)-0.5
$$

and

$$
\chi(x)=\psi^{2}(x)-\frac{1}{12}
$$

The assumptions are true for the Lévy-Smirnov distribution [8], since $\psi$ is monotone increasing and bounded and $\chi$ is bounded and negative at 0 .

Thus it remains to derive the asymptotic covariance matrix $\Sigma=C^{-1} S\left[C^{-1}\right]^{T}$. Here

$$
C=\left(\begin{array}{cc}
E\left(\frac{\partial}{\partial \mu} \psi\left(\frac{\xi-\mu}{\sigma}\right)\right) & E\left(\frac{\partial}{\partial \sigma} \psi\left(\frac{\xi-\mu}{\sigma}\right)\right) \\
E\left(\frac{\partial}{\partial \mu} \chi\left(\frac{\xi-\mu}{\sigma}\right)\right) & E\left(\frac{\partial}{\partial \sigma} \chi\left(\frac{\xi-\mu}{\sigma}\right)\right)
\end{array}\right)
$$

and with random variable $\eta$, it has probability distribution $G_{0}$, where $\mu=0$ and $\sigma=1$,

$$
S=\left(\begin{array}{cc}
E\left(\psi^{2}(\eta)\right) & E(\psi(\eta) \chi(\eta)) \\
E(\psi(\eta) \chi(\eta)) & E\left(\chi^{2}(\eta)\right)
\end{array}\right)
$$

These terms can be evaluated by partial integration. The approximations made by program package Maple 15

$$
C=-\frac{1}{\sigma}\left(\begin{array}{cc}
\frac{1}{2 \pi} & 0 \\
0 & \frac{1}{2 \pi^{2}}
\end{array}\right)
$$


and

$$
S=\left(\begin{array}{cc}
\frac{1}{12} & 0 \\
0 & \frac{1}{180}
\end{array}\right)
$$

Comparing the asymptotic variances of the PIT estimators $T_{n}$ and $s_{n}$ with the ML estimators $\hat{\mu}$ and $\hat{\sigma}$, respectively, we derive the asymptotic relative efficiencies (ARE). It is known that the ML estimators are asymptotically efficient, having also an asymptotic normal distribution, with covariance matrix denoted by $\Sigma$.

For the robustness, we easily derive the breakdown point $\varepsilon^{*}\left(T_{n}\right)$ of the location estimator. We find that

$$
\varepsilon^{*}\left(T_{n}\right)=0.5 \text {. }
$$

This is the maximum for PIT estimator.

Because the function $\chi$ is symmetric we can use directly the result of Huber [6] on the breakdown point $\varepsilon^{*}\left(s_{n}\right)$.

Theorem 3. If we choose the distribution $G_{0}$ with $\mu=0$ and $\sigma=1$ for the functions $\psi$ and $\chi$ then the breakdown point $\varepsilon^{*}\left(s_{n}\right)=\frac{1}{3}$. This is the maximum for PIT estimators.

We see that these properties are not similar to ML estimators because the function $\psi$ is redescending.

\section{NUMERICAL ALGORITHM}

We propose an algorithm for estimating the location and the scale simultaneously. Let the general system be

$$
\begin{gathered}
\sum_{i=1}^{n} \psi\left(\frac{\xi_{i}-T}{s}\right)=0, \\
\sum_{i=1}^{n} \chi\left(\frac{\xi_{i}-T}{s}\right)=0
\end{gathered}
$$

where the functions $\psi$ and $\chi$ are given by (1.2).

Step 1: Preestimation of location and scale by $\max \left\{\xi_{i}\right\}$ and median absolute deviation $(M A D)$, i.e.,

$$
T_{n}{ }^{(0)}=\max \left\{\xi_{i}\right\} \quad \text { and } \quad s_{n}{ }^{(0)}=M A D\left\{\xi_{i}\right\}
$$

Step 2: Estimation of location by

$$
T_{n}^{(m+1)}=T_{n}^{(m)}+\frac{s_{n}^{(m)} \sum_{i=1}^{n} \psi\left(\frac{\xi_{i}-T_{n}^{(m)}}{s_{n}^{(m)}}\right)}{n} .
$$


Step 3: Estimation of scale by

$$
\left[s_{n}{ }^{(m+1)}\right]^{2}=\frac{12}{(n-1)} \sum_{i=1}^{n} \psi^{2}\left(\frac{\xi_{i}-T_{n}{ }^{(m+1)}}{s_{n}{ }^{(m)}}\right)\left[s_{n}{ }^{(m)}\right]^{2} .
$$

Step 4: Stop or goto step 2.

Since the function $G_{0}(x)$ is an absolute continuous distribution function, the convergence of this iterative method follows from by Huber's result [6] Section 7.8, if $G_{0}(x)$ is stricly monotone increasing.

\section{Simulation}

The asymptotic results show that the PIT estimators are less efficient than the ML ones. But the efficiency for finite samples might be quite different. Therefore, we simulated finite samples of size $n$ from the Cauchy distribution with $\mu=0$ and $\sigma=1$. From these samples the different estimators were calculated. From $n=500$ and 1000 simulations the mean and the standard deviation were derived for each estimator, which were used for the comparison of their finite sample behaviour. We calculated the correlations of the paired estimators and the mean square error (MSE), too. These show that in pairwise comparison the behaviour of PIT amd ML estimators are rather similar in finite. This is also indicated by their correlations.

\section{REFERENCES}

[1] D. D. Boos and R. J. Serfling, "A note on differentials and the CLT and LIL for statistical functions, with application to M-estimates," Ann. Stat., vol. 8, pp. 618-624, 1980.

[2] L. Csernyák, "On the most frequent value and cohesion of probability functions," Acta Geodaet., Geophys. et Mont. Acad. Sci. Hung., vol. 8, pp. 397-401, 1973.

[3] S. Fegyverneki, "A special joint estimation of location and scale with applications," Publ. Univ. Miskolc, Ser. D, Nat. Sci., Math., vol. 39, pp. 21-27, 1999.

[4] S. Fegyverneki, "Robust estimators and probability integral transformations," Math. Comput. Modelling, vol. 38, no. 7-9, pp. 803-814, 2003.

[5] F. R. Hampel, E. M. Ronchetti, P. J. Rousseeuw, and W. A. Stahel, Robust statistics. The approach based on influence functions, ser. Wiley Series in Probability and Mathematical Statistics. Probability and Mathematical Statistics. New York: John Wiley \& Sons, 1986.

[6] P. J. Huber, Robust statistics., ser. Wiley Series in Probability and Mathematical Statistics. New York: John Wiley \& Sons, 1981.

[7] F. Nagy, "Parameter estimation of the Cauchy distribution in information theory approach," Journal of Universal Computer Science, vol. 12, pp. 1332-1344, 2006.

[8] M. M. Rao and R. J. Swift, Probability theory with applications, 2nd ed., ser. Mathematics and its Applications (Springer). New York: Springer, 2006, vol. 582.

[9] F. Steiner, "Most frequent value procedures (a short monograph)," Geophysical Transactions, vol. 34, pp. 139-260, 1988.

[10] F. Steiner, A geostatisztika alapjai. Budapest: Tankönyvkiadó, 1990. 
Author's address

S. Fegyverneki

University of Miskolc, Department of Applied Mathematics, 3515 Miskolc-Egyetemáros, Hungary

E-mail address: matfs@uni-miskolc.hu 